\title{
A SYSTEM MODEL FOR LIFECYCLE MONITORING OF BRIDGES
}

\author{
Gergő Dori, PhD Candidate, gergo.dori@tum.de \\ Matthias Wild, PhD Candidate, matthias.wild@tum.de \\ André Borrmann, Professor, andre.borrmann@tum.de \\ Oliver Fischer, Professor, oliver.fischer@tum.de \\ Faculty of Civil, Geo and Environmental Engineering
}

\begin{abstract}
In current bridge maintenance practice, condition grades are assigned to individual bridges, based on regularly performed inspections. One of the main limitations to this approach is the subjective nature of grade assignment. To overcome this drawback, major bridge authorities are developing new methods for condition assessment based on collecting and evaluating sensor data. A major challenge in this context is to correctly model the impact of local deteriorations on the entire bridge's state. In this research, a system model-based approach has been developed to accurately model the correlations between the deterioration mechanisms and the measurement values indicating the progress of the deterioration. In addition, the system model describes the impact of the condition of individual bridge components on the condition of the overall bridge system. To this end, the bridge is hierarchically decomposed into modules, components and subcomponents, taking the structural system and mutual dependencies into account. The system model consists of three levels: The lowest level provides elements for modelling the input parameters provided by sensors or manual measurements. The mid-level models the deterioration mechanisms, taking the output of the parameter level into account. The top-most level models the structure of the bridge in a hierarchical manner, starting at the element parts up to the complete bridge system. The bridge's condition is determined by state propagation mechanisms on the basis of logical elements connecting the aforementioned elements. In the end, the system model can be used to simulate the propagation of conditions assignments from the leaves (the sensors) to the top (the entire bridge). The developed system model approach is based on the application of the Systems Modelling Language (SysML). The paper will discuss in detail the advantages and limitations of the developed method and present a number of ostensive examples.
\end{abstract}

Keywords: bridge maintenance, system model, deterioration mechanism, impact tree, logical connections

\section{INTRODUCTION}

In most industrialized countries, large parts of the infrastructure were erected during the 1960's and 1970's. As a result they are now facing an increasingly aging stock of infrastructure buildings. To maintain the infrastructure safe and at the same time to keep the impact on public budget at a tolerable level, an elaborate management scheme for these buildings, including inspections, maintenance and repairs is necessary.

In current bridge maintenance practice, condition grades are assigned to individual bridges, based on regularly performed inspections. One of the main limitations of this approach is the subjective nature of grade assignment, resulting in significantly diverging grades for bridges in the same condition. To overcome this problem, major bridge authorities are developing new methods for condition assessment, based on collecting and evaluating sensor data. A major challenge in this context is to correctly model the impact of local deterioration on the state of the entire bridge.

In this paper we introduce a system-model based approach which is used to precisely model (1) the correlations between the deterioration mechanisms and the measurement values indicating the progress of the deterioration, and (2) the impact of the condition of individual bridge components on the condition of the overall 
bridge system. The resulting impact tree can be used for simulating deterioration mechanisms as well as for determining the actual condition of a bridge, based on sensor measurements. To this end, condition assignments are propagated from the leaf nodes (the sensor measurements) to the top node (the entire bridge). A major function of this propagation mechanism is provided by the logical connection elements which are located between the different layers of the impact tree and model rules for state propagation. These rules can either be based on empirical models or by means of probabilistic or deterministic approaches.

\section{THE SYSTEM MODEL APPROACH}

The key idea of the presented approach is to generate a system model which takes into account dependencies of structural components as well as interactions between deterioration mechanisms. It is important to avoid "blackbox" systems in order to obtain a better understanding of deterioration processes and their interactions (Neumann and Haardt 2012). The developed system model is able to identify causes of damage and to determine the relevance of the damage as well as their impact on individual bridge components as well as the entire bridge system.

The system model approach is described using the example of a deterioration mechanism: corrosion of the reinforcement in a box girder bridge. The degree of damage can be estimated by measuring causes and symptoms. Damage causes can be evaluated in this example by measuring the chloride or moisture content in concrete. Concrete spalling due to an increase in volume by corrosion processes can serve as a symptom for propagated corrosion of reinforcement. As a result, the impact of the corrosion process on the condition of individual bridge elements and the overall bridge system will be determined on the basis of the structural system. An impact on an individual bridge element could be a reduction of the load bearing capacity in the appropriate cross section, for example. For the entire bridge, the consequences depend first of all on the static system, the materials used and some further parameters like loads or environmental impacts. A high degree of damage can result in a loss of serviceability or even load-bearing capacity.

\subsection{The impact tree}

Some system modeling methods are already used to describe real bridge structures (Sianipar and Adams 1997 and LeBeau and Wadia-Fascetti 2000). These approaches have in common that the investigated bridge is subdivided into single structural components, which are linked by logical conjunctions to obtain the entire bridge system model. The level of detail for a structural component depends on the relevance of the element for the structure. Commonly used modeling approaches in the context of reliability analyses are reliability block diagrams, fault tree analysis and event tree analysis (Darmawan and Stewart 2007, Reay and Andrews 2002, Hadipriono et. al. 1986).

In the abovementioned modeling approaches, the states "in service" or "default" can be assigned to individual components. This binary description forms the basis for applying Boolean operators for modeling the interaction between the components. The relevance of each element depends on the particular problem (stability, durability etc.) and system structure. The individual elements of the resulting system model are accordingly linked to each other by means of logical connections. Complex systems can be modeled in this way as serial or parallel system or a combination of the two. The systems structure function can be set up using state variables of individual elements and based on this function, the failure probability of the system can be evaluated.

However, the reduction of complex interrelationships to a binary notation leads to a significant limitation in system modeling of bridge structures. It is not possible to capture partially deteriorated or damaged structural elements in this model. In addition, time dependent modeling of damage processes (e.g. corrosion of reinforcement) are not taken into consideration by the abovementioned approaches.

As mentioned earlier, the key idea of the new concept is to create a system model that describes both structural interdependencies and interactions between structural damage and failures. Using such a model it is possible to determine the causes of a failure and the relevance of the damage, and to determine the effect of the damage on single structural components and on the complete system. Similar to the life-cycle management system proposed by Lukas et. al. (2009) for reinforced concrete buildings the impact tree model introduced here 
enables the analysis of preconditions and the potential of a failure for both single structural components and complete systems. The impact tree is an improvement of the above described fault tree. It uses a more comprehensive rating system and more flexible interconnection elements than those used in the binary condition states of the fault tree (Reay and Andrews 2002)

\subsubsection{The structure level}

The term "system" for the new modeling approach comprises all structural aspects of the artificial structure like the construction method with respect to the static system, the relevant structural components and, on the lowest level, the materials. These components are labeled as structural elements of the bridge and are displayed with solid boxes within the impact tree model. The bridge element itself is identified as the top element of the complete system.

In the course of the structure level preparation the bridge element is fragmented into functionally smaller and smaller structural elements and ordered into a hierarchical structure according to their role in the system of the bridge (see Figure 1).

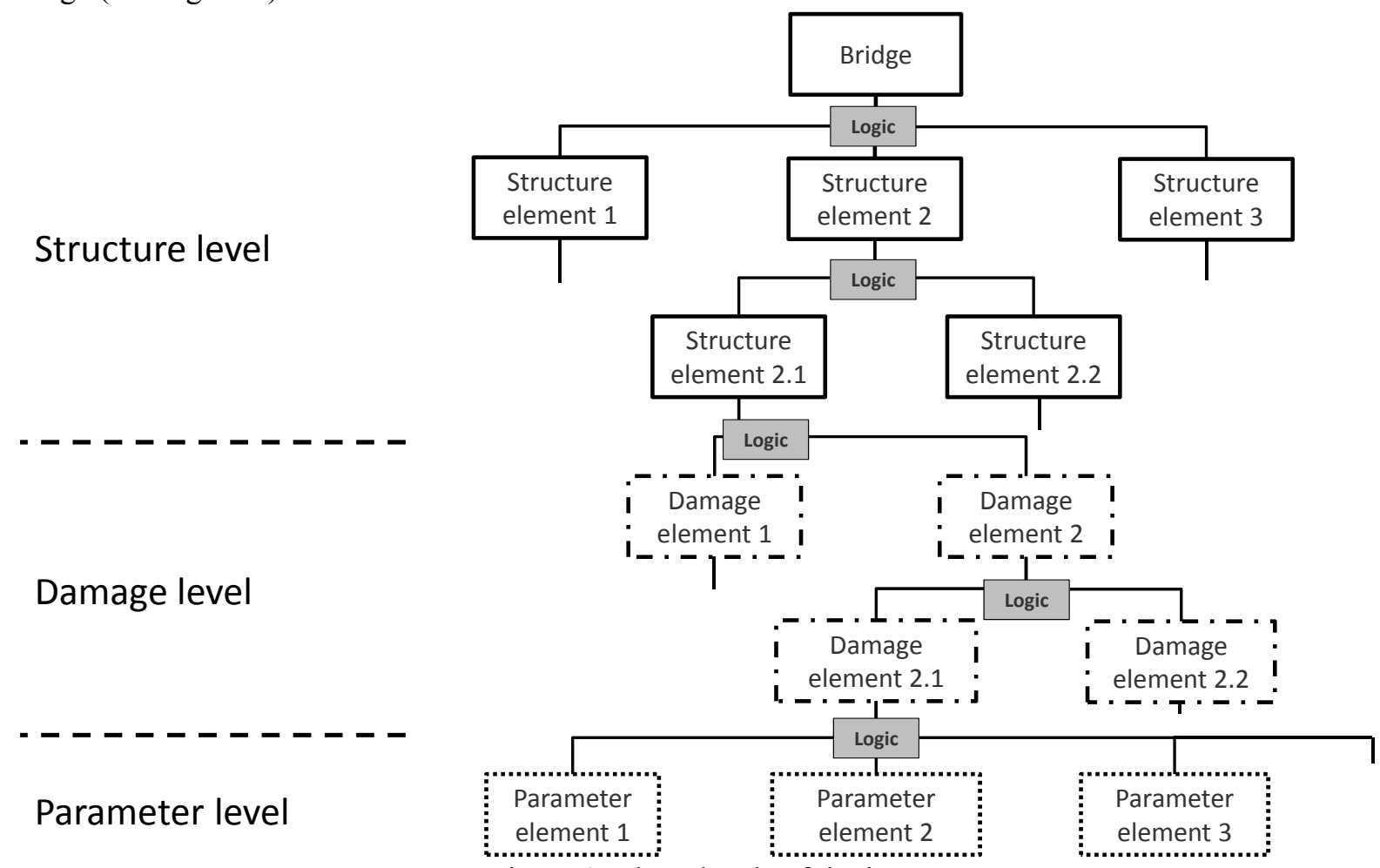

Figure 1: Three levels of the impact tree

\subsubsection{Damage level}

The next level, which extends the structure level, is the damage level. Here, possible damage elements that could occur on a structural component are allocated to the corresponding (lowest) elements of the structure level. A damage element could be e.g. a failure of the reinforcement. The allocation of a damage element to a structural element defines the exact location of the damage.

Damage elements which may have more than one cause will be hierarchically subdivided into further damage elements. As an example the failure of the reinforcement can be subdivided into the elements overload and fatigue (see Figure 2). Damage elements are visualized by dash-dotted boxes within the impact tree models. 


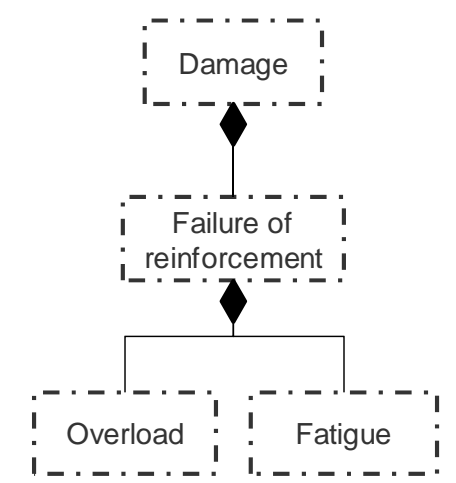

Figure 2: Hierarchic structure of the damage level

\subsubsection{Parameter level}

A further subdivision of the damage elements leads to the finest level of the impact tree: the parameter level. Parameters, which constitute an indication of the beginning or the further propagation of damage, will be allocated to the corresponding lowest element of the damage level. They can either represent a cause or a precondition for a damage. Based on this, the elements either signal the occurrence of damage or an indication that describes the propagation of the damage. For reinforcement corrosion one precondition parameter is the high humidity of the environment. In contrast, the spalling of the concrete surface can already indicate onset or progression of the reinforcement corrosion. All these parameters provide input values for the system analysis.

Wherever possible, all necessary parameters should be a part of a suitable monitoring concept that can feed into the system model real time data to determine the condition of the bridge. Another approach to provide input data for the system model is to manually define the necessary or missing parameters. Although the parameters within the system model are linked to a damage element and thus bounded to a structural element, their location should not be on the corresponding structural element. For example, the settlement of a pier can provide information about the condition of the superstructure elements. Therefore, the measuring process actually takes place on the pier, but the impact is considered at the superstructure element. Parameter elements are depicted as dotted boxes within the impact tree models (see Figure $1 \& 3$ ).

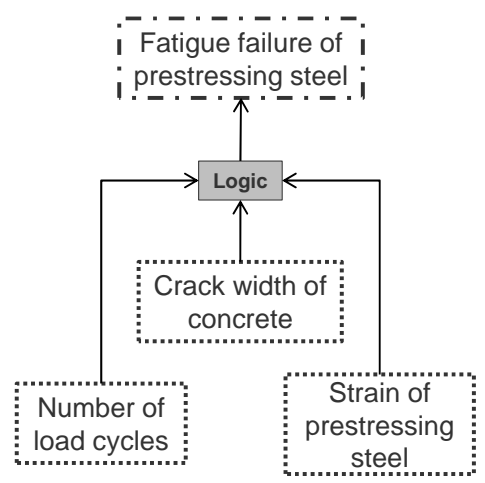

Figure 3. Parameter elements connected with a damage element

\subsection{Interconnections}

A difficulty in creating a correct impact tree is that today there is only a very limited number of reliably described damage interaction processes available. Though there are processes whose possible damage interactions with 
other processes have been highly researched and described (Sudret 2010 and Phetkaysone et. al. 2009), however, the complete scope of all the damage interactions cannot be represented using these models.

For selected system models, interconnections between elements can be described either by probabilistic or by limited value approaches. The biggest difference between the impact tree and the fault tree is precisely these interconnections called logical connection elements. In contrast to the Boolean interconnections of the fault tree (Reay and Andrews 2002), the logical element of the impact tree can describe different and flexible relationships between elements. These relationships can be defined as a combination of equations and rules that describe the propagation of damage from one element to another.

Without any detailed background knowledge about the damage processes, empirical models can be used to create a decision matrix for the model using empirical values or statistical analysis. Input parameters of the matrix are defined by their meaning for the damage process or by their impact on the structure element. These relationships, which are defined in the matrix, determine the output values of the logical elements and describe the degree of damage or condition of a structure element. Statistical results of large series of measurements can be used as useful tool for the development of empirical models for unknown damage processes. The relationships, mapped in the matrix are classified into categories between the values "critical" and "insignificant". Hence, e.g. the impact of chloride concentration can be connected with different structure elements (e.g. foundation, prestressed concrete etc.) and so different statements can be made regarding the degree of damage in the structural component.

If no calculation models or adaptive descriptions of the relationship between damage components and damage propagation are available, the logical elements can be defined by means of the grade assignment methodology representing the current best-practice in bridge inspection. Thus, a stepwise transition from the current approach to a full reliability based procedure can be put into practice (Straub 2009).

\subsection{Modelling the impact tree}

For the modeling of the impact tree a general-purpose graphical modeling language Systems Modeling Language (SysML) is used. It represents a subset of the Unified Modeling Language (UML) with extensions for Systems Modeling applications. While UML has been developed for designing and analyzing software systems, SysML can be used for specifying, analyzing, designing and verifying systems in general (Friedenthal et. al. 2009). By means of parametric diagrams introduced with SysML not only the modeling, but also the simulation of complex systems becomes possible. Providing these features, the language is very well suited for creating a comprehensive bridge model which allows to simulate different deterioration mechanisms and to determine the condition of individual bridge components and the overall bridge. The structure of the impact tree is implemented by means of Block Definition Diagrams which in turn are used by a Parametric Diagram which is used for simulating deterioration processes. How these diagrams are used to run a simulation with the impact tree is introduced in the next chapter.

\section{CONDITION EVALUATION BY THE IMPACT TREE}

The condition of individual system components can be represented by means of a rating scale. For example a tenstage scale or a scale of warning colors similar to traffic lights (green: insignificant, yellow: warning, red: critical) can be implemented for indicating the condition of an element in the impact tree. The condition is determined by means of discrete lower or upper limit values, such as the minimum concrete cover, for example. The classification of the condition on a scale is independent of the calculation rule implemented in the logical element. Thus a consistent format can be used to show the condition of all elements.

The process using of condition evaluation traverses the impact tree from bottom to top. The input values of parameter elements are checked and updated constantly during the evaluation. In the from above connected logical operation, these values are evaluated by predefined calculation rules. The simplest calculation rule is a comparison with value limits. If the values are below the limit, the rating scale shows an insignificant state (green light). Exceeding predetermined limit values leads to a warning or critical state based on the difference between the limit and the measuring values. The damage element will be activated if a signal of the subordinate parametric 
element shows a warning or critical value. Also, the logical element between damage and structure level of this branch will be activated. The rating scale of the structure element gives notice of its condition which is calculated by the connected logical element. The condition evaluation process continues in loops until the parameter values change and an uncritical state is reached or until the top element in the structure level (which symbolizes the bridge itself) is reached.

The use of an impact tree is presented using the example of a single span reinforced concrete bridge (presented in Figure 4).

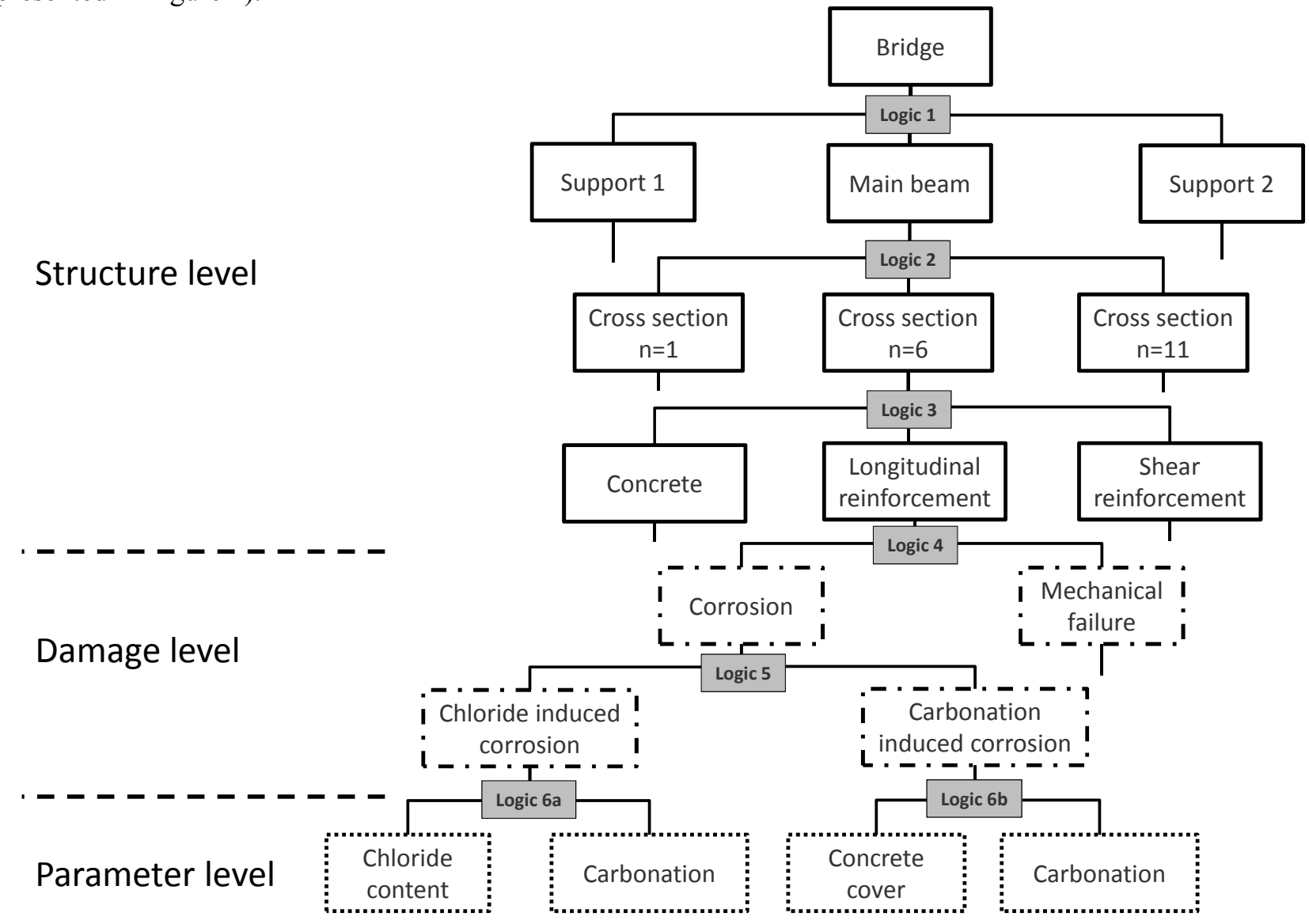

Figure 4: Impact tree of the introduced example

\subsection{Deterioration mechanisms}

As a representative deterioration mechanism corrosion of longitudinal reinforcement is chosen in this example. In the damage level of the impact tree (Fig. 4) the sub-elements "chloride induced corrosion" and "carbonation induced corrosion" are representing the cause of damage.

In this example an approach by Novak et al. (2002) was selected to model the deterioration mechanisms. This approach is implemented in the connection elements "logic 6a" and "logic $6 \mathrm{~b}$ " of the impact tree in form of a decision matrix. The incorporated input values are carbonation, concrete cover and chloride content (Tab. $1 \& 2$ ). 
Tab. 1: Corrosion rate due chloride induced corrosion 0

\begin{tabular}{|c|c|c|}
\hline Carbonation & $\begin{array}{l}\text { Chloride content } \\
\mathrm{M} \%\end{array}$ & Corrosion rate $\mu \mathrm{m} / \mathrm{a}$ \\
\hline \multirow[t]{2}{*}{ yes } & 0,5 & 100 \\
\hline & 2,0 & 150 \\
\hline \multirow[t]{2}{*}{ no } & 0,5 & 10 \\
\hline & 2,0 & 50 \\
\hline
\end{tabular}

Tab. 2: Corrosion rate due carbonation induced corrosion 0

\begin{tabular}{|c|c|c|}
\hline Carbonation & $\begin{array}{l}\text { Concrete cover d } \\
\mathrm{cm}\end{array}$ & Corrosion rate $\mu \mathrm{m} / \mathrm{a}$ \\
\hline yes & $\begin{array}{c}d \leq 2 \\
2<d \leq 4 \\
d>4\end{array}$ & $\begin{array}{l}60 \\
20 \\
30\end{array}$ \\
\hline no & - & 0 \\
\hline
\end{tabular}

\subsection{Structure of the impact tree}

In any case, the root element of an impact tree represents the structure as a whole - in this example the overall bridge. In the next division the bridge is subdivided according to its static system into the supports and the main beam. Since the condition of the main beam is the focus of this example, it is modeled as a combination of several cross sections. For each of them, the lowest partition of the structure level is built of the used materials (concrete, longitudinal and shear reinforcement).

By means of the element "logic 4" structure and damage level are connected. Since in this case study corrosion processes of longitudinal reinforcement are discussed, the remaining branches of the impact tree are neglected. According to the used approach of modeling the corrosion process, associated damage elements (chloride and carbonation induced corrosion) with their appropriate parameter elements are modeled.

The elements of the impact tree are linked with each other by means of connection elements (logic 1-6) as shown in Figure 4. The resulting impact tree can be used for propagating the condition from the parameter level to the root element. In the following the main logical connection elements are specified.

Logical connection elements are modeled as Constraint Blocks, which are particular elements of the SysML Block Definition Diagram class that can be used to define mathematical formulas for the element. These elements contain input and output parameters and an Element Script that formulates the function of the constraint block and defines the executable components of the constraints.

The logical elements $6 \mathrm{a}$ and $6 \mathrm{~b}$ of the impact tree determine the state and criticality of the chloride and carbonation induced corrosion with the appropriate corrosion rate based on the decision matrices of Tab. 1 and Tab. 2, respectively. To this end, they make use of two corresponding input parameter (see Fig. 4) and the Element Script of the logical elements. For Example the following Element Script of "logic 6a" describes the decision matrix of Tab. 1 (in JavaScript): 


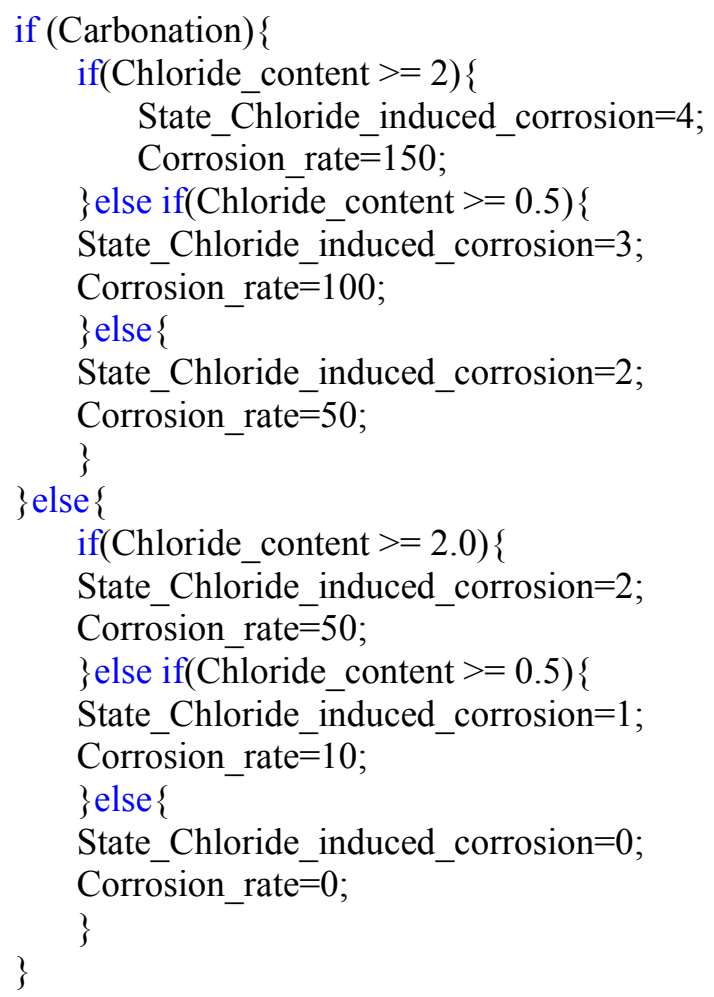

As input parameter the Carbonation and the Chloride_content is used in order to determine the Corrosion_rate and the State_Chloride_induced_corrosion (criticality of the corrosion).

In the next step, in "logic 5", the interaction of both damage mechanisms is determined. In the current example the processes are assumed as accumulative. The remaining cross section of reinforcement is calculated in "logic 4" in consideration of the determined corrosion rate and a certain time period. In "logic 3" the statically required reinforcement is calculated and compared to the remaining cross section of reinforcement. Depending on this comparison the condition of the structure element (cross section $n$ ) is shown on the connected rating scale. Due to the fact that the system is statically determined, the condition of the worst rated cross-section is identical to the condition of the main beam and the entire structure. When all these rules and functions are implemented as Element Scripts for the corresponding logical element (Constraint Block) within the impact tree, then a simulation or analysis of the system model becomes possible.

\subsubsection{Analysis by the impact tree}

The impact tree system model facilitates the analysis of deterioration processes and causes in the examined structure.

One option for using the impact tree for analysis tasks is to determine structural hot-spots. To realize this, parameter elements are kept constant at a certain default value, while the structural element under investigation (main beam) is subdivided in a certain number of cross sections. To get a continuous significant curve in the result diagram, we introduce a variable longitudinal coordinate $\mathrm{x}$. The corrosion rate is independent of the longitudinal coordinate $\mathrm{x}$, because the input values of all connection elements of parameter and damage level are equal. So the critical cross section depends only on the load applied on the main beam. Assuming a uniformly distributed load, the bending moment in the middle is at its maximum. A comparison of the required to the remaining reinforcement by the implemented calculation rule leads to the result that the middle part of the beam is identified as most critical. This method easily can be transferred to more complex structures.

The prediction of future condition states is another possible application of an impact tree. A precondition is here a time dependent definition of deterioration processes. In the example the corrosion process is time dependent due to the corrosion rate in micrometer per year. The variation of the input parameter time results in 
time-condition diagrams of all elements. By means of this result the critical moment of structure elements or the bridge as a whole can be determined.

The influence of different damage mechanisms or some measuring values can also be investigated by the impact tree. For this application parameter elements of interest can be varied. The consequences of parameter variation and thus the impact of the parameter on the element's condition is indicated by the reaction of the rating scale. In the given example, a variation of chloride concentration with or without present carbonation is a suitable parameter to determine the influence of measurement values (parameters) on the deterioration process.

The determination of the actual condition of the structure is one of the most important applications of the impact tree. In combination with an appropriate monitoring system the condition assessment can be executed in real time. Measuring devices applied at identified hot-spot areas deliver the input values for the system analysis by the impact tree. However, also additional locations have to be taken into account when planning the monitoring concept. Due to the fact that each element of the impact tree, whether structure element, damage element or parameter element, is connected to a rating scale which is representing its condition, it is not only possible to determine the condition of the bridge itself; in addition causes of damage (parameter level) and the propagation of damage can be identified.

\section{CONCLUSION}

In this paper we introduced a novel system-model based approach for determining the condition of bridges, based on sensor measurements. The impact tree consists of three different levels (structure level, damage level and parameter level), and allows precise modeling of (1) the correlations between the deterioration mechanisms and the measurement values indicating the progress of the deterioration, and (2) the impact of the condition of individual bridge components on the condition of the overall bridge system.

There are different possible applications of the impact tree. It can be used to simulate deterioration mechanisms by variation of parameter elements as input values during the planning phase of a bridge to detect critical damage mechanisms or structural components - so-called hot-spots. Also it is possible to determine the actual condition of a bridge, based on sensor measurements in real time. On the basis of this information the impact tree can be used as well to determine future condition states by varying significant input values. For all applications, condition assignments are propagated from the leaf nodes (the sensor measurements) to the top node (the entire bridge). A major function in this propagation mechanism is provided by the logical connection elements which are introduced between the different layers of the impact tree and model rules for state propagation. In contrast to the Boolean interconnections of the conventional fault tree, these logical elements are able to describe more complex relationships between elements using flexible combination of equations and rules that describe the propagation of damage from one element to another.

Thanks to its numerous advantages, the impact tree represents the next generation of modelling approaches for bridge maintenance problems. New investigations of damage mechanisms can be implemented easily in the logical connection elements of an impact tree. Future work will concentrate on developing logical connection elements for a broad range of deterioration mechanisms and different approaches to condition assessment (deterministic / probabilistic).

\section{Acknowledgment}

This report is based on parts of the research project carried out at the request of the Federal Ministry for Transport, Construction and Town Planning, represented by the Federal Highway Research Institute, under research project No. FE-Nr. 15.0538 / 2011/ARB. Exclusively the authors are responsible for the content of this manuscript. 


\section{References}

Darmawan, M., S., Stewart, M., G., (2007). "Spatial time-dependent reliability analysis of corroding pretensioned prestressed concrete bridge girders". Structural Safety, Volume 29, Issue 1, 16-31.

Friedenthal, S., Moore, A., Steiner, A., (2008). "A practical guide to SysML: the systems modeling language". Elsevier/Morgan Kaufmann, Boston.

Hadipriono, F., Lim, C., Wong, K., (1986). "Event tree analysis to prevent failures in temporary structures". Journal of Construction Engineering and Management, 112 (4), 500-513.

LeBeau, K., H., Wadia-Fascetti, S., J., (2000). "A fault tree model of bridge deterioration”. Specialty Conference on Probabilistic Mechanics and Structural Reliability.

Lukas, K., Borrmann, A., Zintel, M., Mayer, T., Rank, E., (2009). "Developing a Life-Cycle Management System for Reinforced Concrete Buildings based on Fully-Probabilistic Deterioration Models". Proc. of the 12th International Conference on Civil, Structural and Environmental Engineering Computing. Madeira, Portugal.

Neumann, T., Haardt, P., (2012). "Intelligent Bridges - Adaptive Systems for Information and Holistic Evaluation in Real Time". Proc. of the 6th European Workshop on Structural Health Monitoring - We.4.C.3 - Dresden, Germany.

Novak, B., Brosge, S., Reichert, F., (2002). „Weiterentwicklung von Verhaltensmodellen im Rahmen des BMS“. Bundesanstalt für Straßenwesen.

Phetkaysone, A., Ishida, T., Sato, R., (2009). "Corrosion Cracks and Deflection of RC Beams Subjected to Salty Water Spray under Sustained Load". 2nd International RILEM Workshop on Concrete Durability and Service Life Planning, 55-62.

Reay, K., A., Andrews, J., D., (2002). "A fault tree analysis strategy using binary decision diagrams". Reliability Engineering \& System Safety, Volume 78, Issue 1, 45-56.

Sianipar, P., R., M., Adams, T., M., (1997). "Fault-Tree Model of Bridge Element Deterioration Due to Interaction". Journal of Infrastructure Systems, No. 3, ASCE Press, 103-110.

Straub, D., (2009). "Stochastic modeling of deterioration processes through dynamic Bayesian networks", Journal of Engineering Mechanics 135(10): 10 89-99.

Sudret, B., (2008). "Probabilistic models for the extent of damage in degrading reinforced concrete structures". Reliability Engineering and System Safety, 93 (3), 410-422. 